\title{
Ansiedad por el aislamiento domiciliario producto del coronavirus y el afrontamiento en el estudiante universitario
}

\author{
Anxiety about home isolation due to the coronavirus and coping in university students \\ Ansiedade com o isolamento domiciliar devido ao coronavírus e enfrentamento em \\ estudantes universitários
}

ARTICULO DE INVESTIGACIÓN

Fabrizio Del Carpio Delgado 1

fdelcarpiod@unam.edu.pe

https://orcid.org/0000-0002-6334-7867

\author{
Bertha Silvana Vera Barrios ${ }^{2}$ \\ bverab@unam.edu.pe \\ https://orcid.org/0000-0002-5378-4895
}

\author{
Rómulo Huacasi Machaca ${ }^{3}$ \\ rhuacasidj@mpfn.gob.pe \\ https://orcid.org/0000-0002-8582-4038
}

${ }^{1}$ Escuela Profesional de Ingeniería Civil, Universidad Nacional de Moquegua, Perú

${ }^{2}$ Escuela Profesional de Ingeniería de Minas, Universidad Nacional de Moquegua, Perú ${ }^{3}$ Instituto de Medicina Legal y Ciencias Forenses Tacna Perú

Recibido 08 de enero 2021 | Arbitrado y aceptado 15 de febrero 2021 | Publicado en 01 abril 2021

RESUMEN

\begin{abstract}
Las universidades de todo el mundo han estado inseguras sobre cuánto tiempo durará la crisis del coronavirus y cómo podría afectar la salud mental de los estudiantes y la facultad. El impacto psicológico ha sido crítico para el aislamiento forzado, creando ansiedad e incertidumbre. Los datos se recolectaron mediante la plataforma virtual de cada estudiante y se evaluó la recolección de información en la Universidad Nacional de Moquegua. Comprender el estado de salud mental de los estudiantes producto del covid19 durante la epidemia a 397 estudiantes de la Universidad Nacional de Moquegua, el método utilizado fue el cuestionario Carver para observar los modos de afrontamiento al estrés, para obtener el comportamiento del individuo ante situaciones difíciles, el coronavirus afecta la salud mental: estos son temas prácticamente inexplorados. Estas son circunstancias sin precedentes, y entendemos que crean estrés, favorecen la angustia y la búsqueda feroz de la adquisición de nuevos conocimientos.
\end{abstract}

Palabras clave: Coronavirus; Aislamiento domiciliario; Ansiedad
ABSTRACT

Universities around the world have been unsure about how long the coronavirus crisis will last and how it could affect the mental health of students and faculty. The psychological impact has been critical to the forced isolation, creating anxiety and uncertainty. The data will be collected through the virtual platform of each student and the collection of information will be evaluated at the National University of Moquegua. To understand the state of mental health of the students as a result of the covid-19 during the epidemic in 397 students of the National University of Moquegua, the method used was the Carver questionnaire to observe the modes of coping with stress, to obtain the behavior of the individual before difficult situations, the coronavirus affects mental health: these are practically unexplored topics. These are unprecedented circumstances, and we understand that they create stress, promote anguish and the fierce pursuit of acquiring new knowledge.

Key words: Anxiety, Coping, home isolation, coronavirus.

\section{RESUMO}

Universidades em todo o mundo não têm certeza sobre quanto tempo durará a crise do coronavírus e como ela pode afetar a saúde mental de alunos e professores. 0 impacto psicológico foi crítico para o isolamento forçado, criando ansiedade e incerteza. Os dados foram coletados por meio da plataforma virtual de cada aluno e a coleta de informações foi avaliada na Universidade Nacional de Moquegua. Para compreender o estado de saúde mental dos alunos em decorrência do covid-19 durante a epidemia em 397 alunos da Universidade Nacional de Moquegua, o método utilizado foi o questionário Carver para observar os modos de enfrentamento do estresse, para obter a comportamento do indivíduo diante de situações difíceis, o coronavírus afeta a saúde mental: são temas praticamente inexplorados. São circunstâncias inéditas e entendemos que elas geram estresse, promovem angústia e a busca feroz pela aquisição de novos conhecimentos.

Palavras-chave: Coronavirus; Isolamento em casa; Ansiedade 


\section{INTRODUCCCIÓN}

La UNESCO estima que el cierre de las instituciones educativas debido a la pandemia está afectando a la mitad de los estudiantes del mundo: 890 millones en 114 países. La enseñanza en línea ahora es una nueva rutina para algunos estudiantes, pero presenta desafíos importantes. No todos los alumnos tienen acceso a este tipo de educación, considerando la desigualdad social en muchas naciones (UNESCO, 2020; FVG, 2020). Según el monitoreo de la UNESCO, más de 160 países implementaron cierres a nivel nacional, lo que impactó a más del $87 \%$ de la población estudiantil del mundo. Varios otros países implementaron cierres escolares localizados; En caso de que estos cierres lleguen a todo el país, millones de estudiantes adicionales experimentarán interrupciones educativas (UNESCO, 2020).

La nueva neumonía coronaria (COVID19) se ha convertido en una emergencia mundial de salud pública. A fines de diciembre de 2019, quedó claro que un nuevo coronavirus se estaba extendiendo por Wuhan, la capital de la provincia de Hubei en China (Zhu et al. 2019). Un grupo de pacientes que habían estado expuestos al virus presentaban síntomas de fiebre, disnea $\mathrm{y}$ neumonía grave ( $\mathrm{Li}$ et al. 2020). El 23 de enero de 2020, el Centro Chino para el Control y la Prevención de Enfermedades (CDC de China) registró 835 casos confirmados por laboratorio de infección por COVID-19 y 26 muertes (Chinese Center for Disease Control and Prevention, 2020).

Las personas con la enfermedad pueden tener síntomas inespecíficos, como tos seca, fiebre, dolores musculares; o exhibe cualquier cosa, desde síntomas hasta neumonía grave (Guan, et al. 2020). Desde entonces, se han informado casos confirmados en más de 150 países, con un estimado de 7000 muertes en todo el mundo en marzo de 2020 (World Health Organization, 2020).

La pandemia de Covid-19 no tiene precedentes como un fenómeno de salud pública debido a la combinación de tres factores, un alto nivel de contagio, tasa de mortalidad y ausencia de vacunas, en este sentido, muchas universidades decidieron suspender las clases presenciales y evacuar a los estudiantes para responder a las preocupaciones cada vez más intensas en torno al COVID-19. Esta acción puede tener consecuencias psicológicas negativas entre los estudiantes universitarios. Por ejemplo, los estudiantes educación superior a menudo experimentan emociones negativas compuestas durante el "cierre" de la universidad. Algunos estudiantes que consideran que el campus es acogedor. Pero albergan sentimientos intensos como la frustración, la ansiedad y la traición. Algunos pueden luchar con la soledad y el aislamiento mientras se refugia en el lugar, debido a desconexiones de amigos y parejas. Para aquellos que reciben servicios de asesoramiento en el campus, ya que no pueden acceder a los servicios de asesoramiento presencial, lo que exacerba sus síntomas psicológicos y aumenta el riesgo de suicidio y abuso de sustancias de algunos estudiantes (Van Bortel et al. 2020).

Los estudiantes universitarios experimentan angustia contribuida por la incertidumbre de la interrupción abrupta del semestre, además de la ansiedad causada por el cierre de la escuela y universidades. A medida que más universidades hacen la transición al aprendizaje remoto después de las vacaciones de primavera, algunos 
estudiantes sufren de mala salud mental debido a la interrupción de la rutina académica (Agnew, Poole, y Khan, 2019).

Muchos estudiantes universitarios han perdido sus trabajos en el campus debido a la evacuación, y la cuestión pendiente de las tarifas de alojamiento y comida puede agravar sus dificultades financieras y los resultados de salud mental. También tienen preocupaciones y temores de infección y transmisión de COVID-19 a sus familiares cuando regresan a sus hogares. Dado que los jóvenes pueden ser portadores asintomáticos, los estudiantes pueden estar preocupados por poner a sus familiares mayores en alto riesgo de infección con complicaciones graves por COVID-19. El hecho de que la pandemia de COVID-19 afecte la salud mental universitaria solo subraya la necesidad urgente de comprender estos desafíos y preocupaciones para informar el desarrollo de cursos de acción y mensajes de salud pública que apoyarán a los estudiantes universitarios durante este momento difícil (Pan, et al. 2020).

Centrarse en la salud psicológica de las personas menores de 18 años y personas de mediana edad a mayores (mayores de 50 años) y proporcionar intervención psicológica dirigida a través de múltiples canales como televisión, Internet y teléfono. Para los menores de 18 años, los departamentos de educación del gobierno, las escuelas y los maestros deben guiarlos para comprender la epidemia correctamente. También deben tratar de aliviar el pánico y el miedo estableciendo una actitud optimista y positiva. Para las personas de mediana edad y mayores de 50 años, el gobierno debería alentar a los niños de este grupo a explicar la ciencia popular de la epidemia y acompañarlos con mayor frecuencia para garantizar una buena salud psicológica (Tian et al. 2020).

La COVID-19 y sus efectos acompañantes continuarán impactando profundamente la salud mental y el bienestar universitario; a consecuencia la salud mental desempeña un papel crucial en la lucha contra la epidemia. Es imperativo para las universidades crear conciencia sobre las necesidades $y$ preocupaciones de salud mental de los estudiantes, y capacitar a sus estudiantes para buscar ayuda y apoyo durante este desastre biológico. Los estudiantes universitarios deben adaptar las estrategias de afrontamiento para satisfacer sus necesidades específicas y promover su capacidad de recuperación psicológica. Los esfuerzos considerables realizados por las universidades deberían dedicarse a ayudar a los estudiantes a prosperar en esta crisis. Con la experiencia obtenida apoyando a los estudiantes en esta pandemia, las universidades estarán bien posicionadas para ayudar a los estudiantes universitarios a mantenerse en mente, cuerpo y espíritu durante otros tiempos difíciles (Zhai, y Du, 2020).

El gobierno de Perú puso fin este martes 30 de junio del 2020 con el DECRETO SUPREMO $\mathrm{N}^{\circ}$ 116-2020-PCM (DECRETO SUPREMO $\mathrm{N}^{\circ}$ 116-2020-PCM., 2020), a una cuarentena nacional de 107 días, con 285,213 contagios acumulados de COVID-19 y 9,677 fallecidos. El miércoles 1 de julio del 2020 acaba el confinamiento para dieciocho de las veinticinco regiones de Perú, quien fue el primer país de Latinoamérica en decretar la cuarentena general, y obligatoria para toda su población el pasado 15 de marzo con DECRETO SUPREMO $\mathrm{N}^{\circ}$ 044-2020-PCM (DECRETO SUPREMO $\mathrm{N}^{\circ}$ 044-2020-PCM., 
2020). Solo siete regiones (Arequipa, Áncash, Ica, Junín, Huánuco, San Martín y Madre de Dios) donde los contagios todavía están en ascenso seguirán oficialmente bajo cuarentena hasta el 31 de julio.

La investigación que se realiza en la Universidad nacional de Moquegua no tiene precedentes para las políticas de reforma educativa ni el seguimiento para la medición del estrés y ansiedad prolongada en los estudiantes universitarios, para esta investigación se realizó el modelo de CAVER para el afrontamiento de la ansiedad y el estrés.

El estrés en términos biológicos como un estado de activación simpático adrenal y neuroendocrino, que se acompaña de inhibición inmunitaria y conductas emocionales negativas como el miedo, indefensión, ansiedad, depresión. Nieto y Nieto (2006). "Es aquel que se produce a partir de las demandas que exige el ámbito educativo" por tal motivo, los estudiantes y profesores se pueden ver afectados por el estrés (Caldera, y Pulido, 2007).

El malestar que el estudiante presenta debido a factores físicos, emocionales, ya sea de carácter interrelacional o ambientales que pueden ejercer una presión significativa en la competencia individual para afrontar el contexto escolar en rendimiento académico, habilidad metacognitiva para resolver problemas, pérdida de un ser querido, presentación de exámenes, relación con los compañeros y educadores, búsqueda de reconocimiento e identidad, habilidad para relacionar el componente teórico con la realidad específica abordada (Martínez y Diaz, 2007).

El estrés académico es un proceso sistémico, de carácter adaptativo y esencialmente psicológico, que se presenta a) cuando el alumno se ve sometido, en contextos escolares, a una serie de demandas que, bajo la valoración del propio alumno son considerados estresores (input); b) cuando estos estresores provocan un desequilibrio sistémico (situación estresante) que se manifiesta en una serie de síntomas (indicadores del desequilibrio); y c) cuando este desequilibrio obliga al alumno a realizar acciones de afrontamiento (output) para restaurar el equilibrio sistémico (Barraza, y Quiñonez, 2007).

El término ansiedad proviene de la palabra del latín anxius que significa agitación. Cuando la ansiedad se basa en miedos racionales y realistas ayuda a manejar situaciones de manera afectiva y estimula a la persona a preservar la vida. Es razonable y apropiado reaccionar con ansiedad ante algunas situaciones de la vida. Pero, según Brourne 1994 y Ellis 2000, si la ansiedad es muy intensa y hace que se pierda el control puede provocar un inadecuado afrontamiento de los problemas, si persiste por mucho tiempo y/o interfiere con la socialización con otros, puede convertirse en un problema de salud (Morales, 2011).

El estrés universitario afecta a variables tan diversas como el estado emocional, la salud física o las relaciones interpersonales. Altos niveles de estresores académicos predicen reacciones de estrés (Misra, Crist, y Burant, 2003). Estudios sobre la repercusión del estrés en la salud de los estudiantes universitarios sugieren que existe una relación entre el estrés y el deterioro del sistema inmunológico y de la salud mental (Bunevicius, Katkute, y Bunevicius, 2008). El estilo de vida de los estudiantes también se ve modificado en los primeros años de estudios y sobre todo según 
se acerca el periodo de exámenes, convirtiéndose así sus hábitos en insalubres (exceso de consumo de cafeína, tabaco, sustancias psicoactivas estimulantes y relajantes, disminución en la práctica de ejercicio físico, nutrición inadecuada) y pudiendo llevar a la aparición de trastornos de salud, disminución de la autoestima y a percepción de peor nivel de salud. Los iguales juegan un papel muy importante en la percepción y reacción al estrés de los estudiantes (Hudd et al. 2000).

\section{METODOLOGÍA}

Esta investigación se basó en un diseño no experimental con un tipo de investigación de campo, como indica Palella Stracuzzi y Martins Pestana (2010), es de diseño no experimental pues es "aquel que se realiza sin manipular en forma deliberada ninguna variable", y es de campo ya que "consiste en la recolección de datos directamente de la realidad donde ocurren los hechos sin manipular o controlar variables". De la misma manera esta investigación posee un nivel descriptivo y explicativo, pues permite la interpretación de la realidad de los hechos asi como permite determinar el origen o las causas de diversos fenomenos dentro de la investigación (Palella Stracuzzi y Martins Pestana, 2010).

La investigación se desarrolló en la ciudad de Moquegua, Perú, comprendido en la Universidad Nacional de Moquegua. Dentro de los materiales empleados para recabar la información de la salud mental de los estudiantes universitarios fue la encuesta de la Metodología de CAVER (Carver, Scheier, y Weintraub, 1989).

La población fue de 1508 estudiantes y se implicó para un tamaño de muestra de 397 estudiantes de la Universidad Nacional de Moquegua. Las Técnicas e instrumentos de recolección de datos usados fueron: el primer instrumento utilizado para la para el estudio fue el Cuestionario COPE, elaborado por Carver, Scheir y Weintraub en 1989, este cuestionario permitió evaluar las formas o estilos de afrontamiento que utiliza la gente ante eventos estresantes, el objetivo del Cuestionario de Modos de Afrontamiento al Estrés (COPE) fue conocer cómo las personas reaccionan o responden cuando enfrentan a situaciones difíciles o estresantes. Con este propósito, Carver, et al. ob. cit. han construido un cuestionario de 52 ítems, que comprenden 13 modos de responder al estrés; los ítems alternativos de cada modo indicó qué cosas hace o siente con más frecuencia el sujeto, cuando se encuentra en tal situación. Este instrumento trató de determinar cuáles son las alternativas QUE MAS USA. Las respuestas se organizaron en una escala de 13 posibilidades, o formas de afrontamiento. Cada una de ellas constó de 4 alternativas o formas de afrontar las cargas de estrés. Los puntajes más altos en la escala indicaban la estrategia de afrontamiento y cual escala era frecuentemente la más empleada.

\section{Preguntas Formuladas.}

\begin{tabular}{llll}
\hline 1 & $\begin{array}{l}\text { Ejecuto acciones adicionales para deshacerme } \\
\text { del problema }\end{array}$ & 27 & Hago paso a paso lo que tiene que hacerse \\
2 & $\begin{array}{l}\text { Elaboro un plan de acción para deshacerme } \\
\text { del problema }\end{array}$ & 28 & $\begin{array}{l}\text { Me pongo a pensar más en los pasos a seguir } \\
\text { para solucionar el problema } \\
\text { Dejo de lado otras actividades para } \\
\text { concentrarme en el problema }\end{array}$ \\
\hline
\end{tabular}




\begin{tabular}{|c|c|c|c|}
\hline 4 & $\begin{array}{l}\text { Me esfuerzo esperando el momento apropiado } \\
\text { para enfrentar el problema. }\end{array}$ & 30 & $\begin{array}{l}\text { Me aseguro de no crear problemas peores por } \\
\text { actuar muy pronto. }\end{array}$ \\
\hline 5 & $\begin{array}{l}\text { Pregunto a personas que han tenido } \\
\text { experiencias similares sobre lo que hicieron }\end{array}$ & 31 & $\begin{array}{l}\text { Hablo con alguien para averiguar más sobre el } \\
\text { problema }\end{array}$ \\
\hline 6 & Hablo con alguien sobre mis sentimientos & 32 & $\begin{array}{l}\text { Converso con alguien sobre lo que me está } \\
\text { sucediendo. }\end{array}$ \\
\hline 7 & Busco algo bueno de lo que está pasando & 33 & Aprendo algo de la experiencia \\
\hline 8 & Aprendo a convivir con el problema. & 34 & $\begin{array}{l}\text { Me acostumbro a la idea de que el problema ya } \\
\text { ha sucedido }\end{array}$ \\
\hline 9 & Busco la ayuda de Dios & 35 & Trato de encontrar consuelo en mi religión \\
\hline 10 & $\begin{array}{l}\text { Emocionalmente me perturbo y libero mis } \\
\text { emociones }\end{array}$ & 36 & $\begin{array}{l}\text { Siento mucha perturbación emocional y } \\
\text { expreso esos sentimientos a otros }\end{array}$ \\
\hline
\end{tabular}

11 Me niego a aceptar que el problema ha ocurrido.

37 Me comporto como si no hubiese ocurrido el problema

12 Dejo de lado mis metas

38 Acepto que puedo enfrentar al problema y lo dejo de lado

13 Me dedico a trabajar o realizar otras actividades para alejar el problema de mi mente

14 Concentro mis esfuerzos para hacer algo sobre el problema

15 Elaboro un plan de acción

39 Sueño despierto con otras cosas que no se relacionen al problema

16 Me dedico a enfrentar el problema, y si es necesario dejo de lado otras actividades

17 Me mantengo alejado del problema sin hacer nada, hasta que la situación lo permita

40 Actúo directamente para controlar el problema

41 Pienso en la mejor manera de controlar el problema

42 Trato que otras cosas no interfieran en los esfuerzos que pongo para enfrentar el problema

43 Me abstengo de hacer algo demasiado pronto

18 Trato de obtener el consejo de otros para saber qué hacer con el problema

19 Busco el apoyo emocional de amigos o familiares

20 Trato de ver el problema en forma positiva

21 Acepto que el problema ha ocurrido y no podrá ser cambiado

22 Deposito mi confianza en Dios

23 Libero mis emociones

Hablo con alguien que podría hacer algo concreto sobre el problema

Busco la simpatía y la comprensión de alguien.

46 Trato de desarrollarme como una persona a consecuencia de la experiencia

$47 \quad$ Acepto que el problema ha sucedido

24 Actúo como si el problema no hubiera sucedido realmente.

25 Dejo de perseguir mis metas

48 Rezo más de lo usual

49 Me perturbo emocionalmente y estoy atento al problema

50 Me digo a mí mismo: "Esto no es real"

26 Voy al cine o miro la TV, para pensar menos en el problema

51 Disminuyo los esfuerzos que pongo para solucionar el problema

52 Duermo más de lo usual. 


\section{Calificación}

Para la calificación se fueron sumados los puntajes obtenidos en cada una de las 13 áreas: Afrontamiento, directo, planificación de actividades, supresión de actividades, retracción de afrontamiento, busca soporte social, busca soporte emocional, reinterpretación, aceptación, retorno a la religión, análisis de emociones, negación, conducta inadecuada y distracción. Al puntaje obtenido se multiplicó por la constante 0.25 . Posteriormente fue ubicado el puntaje hallado en el Perfil considerando que dicho perfil fue representado en porcentaje (\%) multiplicando el puntaje obtenido por 10 .

El segundo instrumento fue la dimensión del inventario de depresión Beck II-(BDI-II), son 21 los indicadores en sus tres dimensiones del inventario de depresión Beck, dividido en tres áreas, área cognitiva, área física /conductual y área afectiva / emocional. La interpretación del intervalo de Beck se determina asignando tres valores a cada uno de los ítems. Los valores de cada elemento se suman obteniendo una puntuación total que puede ir de 0 a 63 puntos. Una puntuación total de 0 a 7 se interpreta como un nivel mínimo de ansiedad, de 8 a 15 como leve, de 16 a 25 como moderado y de 26 a 63 como severa.

\section{Procesamiento de datos}

El procedimiento de recogida de datos se hizo de forma colectiva y virtual, realizando previas coordinaciones con el área de informática de la universidad nacional de Moquegua, los datos fueron luego procesados estadísticamente.

\section{RESULTADOS Y DISCUSIÓN}

La información directa, rápida y mediata con la encuesta virtualizada de la Metodología de CAVER Carver, Scheier, y Weintraub (1989), conllevó a procesar y obtener resultados que en primer lugar se muestra en la Tabla 1 las generalidades que pertenecen los estudiantes universitarios de la región de Moquegua 2020.

Tabla 1. Generalidades.

\begin{tabular}{|c|c|c|}
\hline $\begin{array}{c}\text { Escuela a la que pertenecen los Estudiantes de la } \\
\text { Universidad de Moquegua }\end{array}$ & №. & $\%$ \\
\hline Gestión pública y desarrollo social & 49 & 12,34 \\
\hline Ingeniería agroindustrial & 39 & 9,82 \\
\hline Ingeniería ambiental & 84 & 21,16 \\
\hline Ingeniería de minas & 165 & 41,56 \\
\hline Ingeniería de sistemas e informática & 46 & 11,59 \\
\hline Ingeniería pesquera & 14 & 3,53 \\
\hline TOTAL & 397 & 100,0 \\
\hline EDAD & №. & $\%$ \\
\hline$<18$ años & 66 & 16,6 \\
\hline $18-28$ & 307 & 77,3 \\
\hline 29-39 & 18 & 4,5 \\
\hline$\geq 40$ & 6 & 1,5 \\
\hline TOTAL & 397 & $100 \%$ \\
\hline
\end{tabular}




\begin{tabular}{|c|c|c|}
\hline $\begin{array}{c}\text { Escuela a la que pertenecen los Estudiantes de la } \\
\text { Universidad de Moquegua }\end{array}$ & №. & $\%$ \\
\hline GENERO & №. & $\%$ \\
\hline Femenino & 195 & 49,12 \\
\hline Masculino & 202 & 50,88 \\
\hline TOTAL & 397 & $100 \%$ \\
\hline ESTADO CIVIL & №. & $\%$ \\
\hline Casado & 10 & 2,52 \\
\hline Soltero & 387 & 97,48 \\
\hline TOTAL & 397 & 100,0 \\
\hline DEPENDE DE OTRO & №. & $\%$ \\
\hline No & 98 & 24,69 \\
\hline$S i$ & 299 & 75,31 \\
\hline TOTAL & 397 & 100,0 \\
\hline
\end{tabular}

En la Tabla 1 se muestra que el $41.56 \%$ de los estudiantes universitarios de la región Moquegua pertenecen a la escuela profesional de Ingeniería de minas, seguido del $12.34 \%$ de estudiantes de la escuela de gestión pública y desarrollo social, mientras que solo el $3.53 \%$ son de ingeniería pesquera, de la misma manera se evidencia que el $77.3 \%$ de los estudiantes universitarios tienen entre 18 a 28 años, seguido del $16.6 \%$ de estudiantes menores de 18 años, el $4.5 \%$ tienen entre 29 a 39 años, mientras que solo el $1.5 \%$ tienen 40 años o más. Igualmente se muestra que el $50.88 \%$ de los estudiantes son de sexo masculino, mientras que el $49.12 \%$ de los estudiantes son de sexo femenino y el $97.48 \%$ de los estudiantes son solteros, mientras que el $2.52 \%$ de los estudiantes son casados. De la misma manera el $75.31 \%$ de los estudiantes universitarios de la región Moquegua dependen de otras personas, mientras que solo el $24.69 \%$ de los estudiantes no dependen económicamente de nadie.

Seguidamente en la Tabla 2 se presenta el grado de ansiedad/estrés a consecuencia de la pandemia en los alumnos de la Universidad Nacional de Moquegua.

Tabla 2. Grado de ansiedad/estrés a consecuencia de la pandemia en los alumnos de la universidad nacional de Moquegua.

\begin{tabular}{ccc}
\hline ANSIEDAD & №. & \% \\
\hline Mínima & 144 & 36,27 \\
Leve & 88 & 22,17 \\
Moderada & 82 & 20,65 \\
Severa & 83 & 20,91 \\
\hline TOTAL & $\mathbf{3 9 7}$ & $\mathbf{1 0 0 , 0}$ \\
\hline
\end{tabular}

La Tabla 2 muestra que el $36.27 \%$ de los estudiantes universitarios de la región Moquegua presentaron niveles mínimos o casi inexistentes de ansiedad, seguido del $22.17 \%$ de estudiantes con ansiedad leve, el 20.91\% presentaban ansiedad severa, mientras que otro $20.65 \%$ ansiedad moderada. 
En la tabla 3 se presentan las estrategias de afrontamiento en los alumnos de la Universidad de Moquegua.

Tabla 3. Estrategias de afrontamiento en los alumnos de la universidad Nacional de Moquegua.

\begin{tabular}{|c|c|c|c|c|c|c|}
\hline \multirow{2}{*}{ AFRONTAMIENTO } & \multicolumn{2}{|c|}{ POCAS VECES } & \multicolumn{2}{|c|}{$\begin{array}{c}\text { DEPENDE DE } \\
\text { CIRCUNSTANCIAS }\end{array}$} & \multicolumn{2}{|c|}{ FRECUENTEMENTE } \\
\hline & №. & $\%$ & №. & $\%$ & №. & $\%$ \\
\hline Afrontamiento directo & 66 & 16,62 & 165 & 41,56 & 166 & 41,81 \\
\hline Planificación de actividades & 72 & 18,14 & 168 & 42,32 & 157 & 39,55 \\
\hline Supresión de actividades & 133 & 33,50 & 162 & 40,81 & 102 & 25,69 \\
\hline Retracción de afrontamiento & 44 & 11,08 & 267 & 67,25 & 86 & 21,66 \\
\hline Búsqueda de soporte social & 122 & 30,73 & 109 & 27,46 & 166 & 41,81 \\
\hline Busca soporte emocional & 161 & 40,55 & 120 & 30,23 & 116 & 29,22 \\
\hline Reinterpretación positiva & 30 & 7,56 & 108 & 27,20 & 259 & 65,24 \\
\hline Aceptación & 27 & 6,80 & 168 & 42,32 & 202 & 50,88 \\
\hline Retorno a la religión & 128 & 32,24 & 191 & 48,11 & 78 & 19,65 \\
\hline Análisis de emociones & 237 & 59,70 & 131 & 33,00 & 29 & 7,30 \\
\hline Negación & 187 & 47,10 & 153 & 38,54 & 57 & 14,36 \\
\hline Conducta inadecuada & 257 & 64,70 & 117 & 29,47 & 23 & 5,79 \\
\hline Distracción & 103 & 25,94 & 231 & 58,19 & 63 & 15,87 \\
\hline
\end{tabular}

En la Tabla 3 se evidenció que el 41.81\% de los estudiantes universitarios de la región Moquegua presentan frecuentemente afrontamiento directo, el $50.88 \%$ suelen presentar con frecuencia el estilo de aceptación, el $40.55 \%$ pocas veces presenta búsqueda de soporte emocional, el 58.19\% presentan distracción dependiendo de las circunstancias, mientras que el $18.14 \%$ pocas veces tienen planificación de actividades.

De la misma manera en la Tabla 4 se evidencia la relación entre el grado de ansiedad/estrés a consecuencia de la pandemia y el estilo de afrontamiento directo en los alumnos de la Universidad Nacional de Moquegua.

Tabla 4. Relación entre el grado de ansiedad/estrés a consecuencia de la pandemia y el estilo de afrontamiento directo en los alumnos de la Universidad Nacional de Moquegua.

\begin{tabular}{|c|c|c|c|c|c|c|c|c|c|c|}
\hline \multirow{3}{*}{$\begin{array}{l}\text { Afrontamiento } \\
\text { directo }\end{array}$} & \multicolumn{8}{|c|}{ ANSIEDAD } & \multirow{2}{*}{\multicolumn{2}{|c|}{ TOTAL }} \\
\hline & \multicolumn{2}{|c|}{ Mínima } & \multicolumn{2}{|c|}{ Leve } & \multicolumn{2}{|c|}{ Moderada } & \multicolumn{2}{|c|}{ Severa } & & \\
\hline & №. & $\%$ & №. & $\%$ & №. & $\%$ & №. & $\%$ & №. & $\%$ \\
\hline Pocas veces & 14 & 3,53 & & 3,78 & 17 & 4,28 & 20 & 5,04 & 66 & 16,62 \\
\hline Dep. circunstancias & 59 & 14,86 & 15 & 9,57 & 36 & 9,07 & 32 & 8,06 & 165 & 41,56 \\
\hline Frecuentemente & 71 & 17,88 & $\begin{array}{l}38 \\
35\end{array}$ & 8,82 & 29 & 7,30 & 31 & 7,81 & 166 & 41,81 \\
\hline TOTAL & 144 & 36,27 & 88 & 22,17 & 82 & 20,65 & 83 & 20,91 & 397 & 100 \\
\hline
\end{tabular}


En base a los resultados mostrados en la Tabla 4, según la prueba de chi cuadrado $\left(X^{2}=11.34\right)$ muestra que el grado de ansiedad $\mathrm{y}$ el afrontamiento directo no presenta relación estadística significativa $(\mathrm{P}>0.05)$. Asimismo se observa que el $17.88 \%$ de los estudiantes universitarios de la región Moquegua con ansiedad mínima presentan frecuentemente afrontamiento directo, mientras que el $5.04 \%$ de los estudiantes con ansiedad severa presentan este tipo de afrontamiento pocas veces.

Seguidamente en la Tabla 5 se muestra la relación existente entre el grado de ansiedad/estrés a consecuencia de la Pandemia y la Planificación de actividades en los alumnos de la Universidad Nacional de Moquegua.

Tabla 5. Relación entre el grado de ansiedad/estrés a consecuencia de la pandemia y la planificación de actividades en los alumnos de la Universidad Nacional de Moquegua.

\begin{tabular}{|c|c|c|c|c|c|c|c|c|c|c|}
\hline \multirow{3}{*}{$\begin{array}{l}\text { Planificación de } \\
\text { actividades }\end{array}$} & \multicolumn{8}{|c|}{ ANSIEDAD } & \multirow{2}{*}{\multicolumn{2}{|c|}{ TOTAL }} \\
\hline & \multicolumn{2}{|c|}{ Mínima } & \multicolumn{2}{|c|}{ Leve } & \multicolumn{2}{|c|}{ Moderada } & \multicolumn{2}{|c|}{ Severa } & & \\
\hline & №. & $\%$ & №. & $\%$ & №. & $\%$ & №. & $\%$ & №. & $\%$ \\
\hline Pocas veces & 20 & 5,04 & 16 & 4,03 & 18 & 4,53 & 18 & 4,53 & 72 & 18,14 \\
\hline Dep. circunstancias & 55 & 13,85 & 38 & 9,57 & 36 & 9,07 & 39 & 9,82 & 168 & 42,32 \\
\hline Frecuentemente & 69 & 17,38 & 34 & 8,56 & 28 & 7,05 & 26 & 6,55 & 157 & 39,55 \\
\hline TOTAL & 144 & 36,27 & 88 & 22,17 & 82 & 20,65 & 83 & 20,91 & 397 & 100 \\
\hline & & $2=8.33$ & & $P>0.05$ & & $P=0.21$ & & & & \\
\hline
\end{tabular}

Es así como en la Tabla 5 aplicando la prueba de chi cuadrado $\left(\mathrm{X}^{2}=8.33\right)$ se muestra que el grado de ansiedad y la planificación de actividades no presenta relación estadística significativa $(\mathrm{P}>0.05)$. Asimismo, se observa que el $17.38 \%$ de los estudiantes universitarios de la región Moquegua con ansiedad mínima frecuentemente presentan el estilo de afrontamiento de planificación de actividades, mientras que el $9.82 \%$ de los estudiantes con ansiedad severa presentan este tipo de afrontamiento dependiendo de las circunstancias.

Posteriormente en la Tabla 6 se muestra la relación existente entre el grado de ansiedad/estrés a consecuencia de la pandemia y la supresión de actividades en los alumnos de la Universidad Nacional de Moquegua.

Tabla 6. Relación entre el grado de ansiedad/estrés a consecuencia de la pandemia y la Supresión de actividades en los alumnos de la Universidad Nacional De Moquegua.

\begin{tabular}{|c|c|c|c|c|c|c|c|c|c|c|}
\hline \multirow{3}{*}{$\begin{array}{l}\text { Supervisión de } \\
\text { Actividades }\end{array}$} & \multicolumn{8}{|c|}{ ANSIEDAD } & \multirow{2}{*}{\multicolumn{2}{|c|}{ TOTAL }} \\
\hline & \multicolumn{2}{|c|}{ Mínima } & \multicolumn{2}{|c|}{ Leve } & \multicolumn{2}{|c|}{ Moderada } & \multicolumn{2}{|c|}{ Severa } & & \\
\hline & №. & $\%$ & №. & $\%$ & №. & $\%$ & №. & $\%$ & №. & $\%$ \\
\hline Pocas veces & 62 & 15,62 & 29 & 7,30 & 20 & 5,04 & 22 & 5,54 & 133 & 33,50 \\
\hline Dep. circunstancias & 50 & 12,59 & 31 & 7,81 & 41 & 10,33 & 40 & 10,08 & 162 & 40,81 \\
\hline Frecuentemente & 32 & 8,06 & 28 & 7,05 & 21 & 5,29 & 21 & 5,29 & 102 & 25,69 \\
\hline TOTAL & 144 & 36,27 & 88 & 22,17 & 82 & 20,65 & 83 & 20,91 & 397 & 100 \\
\hline
\end{tabular}


En base los resultados expresados en la Tabla 6 se aplica la prueba de chi cuadrado $\left(X^{2}=13.92\right)$ muestra que el grado de ansiedad y la supresión de actividades presenta relación estadística significativa $(\mathrm{P}<0.05)$. Según la correlación de Spearman $(\mathrm{Rho}=.415)$ existe una correlación moderada entre las variables. Asimismo, se observa que el $15.62 \%$ de los estudiantes universitarios de la región Moquegua con ansiedad mínima pocas veces presentan el estilo de afrontamiento de supresión de actividades, mientras que el 10.33\% de los estudiantes con ansiedad moderada presentan este tipo de afrontamiento dependiendo de las circunstancias.

\begin{tabular}{ccccc}
\hline & & Correlaciones & & \\
& & Ansiedad & Supresión de actividades \\
\hline Rho de & Ansiedad & Coeficiente de correlación & 1,000 &, $415^{*}$ \\
Spearman & & Sig. (bilateral) & $\cdot$ &, 022 \\
& & N & 397 & 397 \\
& Supresión de & Coeficiente de correlación &, $315^{*}$ & 1,000 \\
& actividades & Sig. (bilateral) &, 022 & $\cdot$ \\
& & N & 397 & 397 \\
\hline
\end{tabular}

*. La correlación es significativa en el nivel 0,05 (bilateral).

Posteriormente en la Tabla 7 se muestra la relación que existió entre el grado de ansiedad/estrés a consecuencia de la pandemia y la retractación de afrontamiento en los alumnos de la Universidad Nacional de Moquegua

Tabla 7. Relación entre el grado de ansiedad/estrés a consecuencia de la pandemia y la retractación de afrontamiento en los alumnos de la Universidad Nacional de Moquegua.

\begin{tabular}{|c|c|c|c|c|c|c|c|c|c|c|}
\hline \multirow{3}{*}{$\begin{array}{c}\text { Retractación de } \\
\text { afrontamiento }\end{array}$} & \multicolumn{8}{|c|}{ ANSIEDAD } & \multirow{2}{*}{\multicolumn{2}{|c|}{ TOTAL }} \\
\hline & \multicolumn{2}{|c|}{ Mínima } & \multicolumn{2}{|c|}{ Leve } & \multicolumn{2}{|c|}{ Moderada } & \multicolumn{2}{|c|}{ Severa } & & \\
\hline & №. & $\%$ & №. & $\%$ & №. & $\%$ & №. & $\%$ & №. & $\%$ \\
\hline Pocas veces & 20 & 5,04 & 4 & 1,01 & & 2,52 & 10 & 2,52 & 44 & 11,08 \\
\hline Dep. circunstancias & 95 & 23,93 & 65 & 16,37 & $\begin{array}{l}10 \\
53\end{array}$ & 13,35 & 54 & 13,60 & 267 & 67,25 \\
\hline Frecuentemente & 29 & 7,30 & 19 & 4,79 & $\begin{array}{l}53 \\
19\end{array}$ & 4,79 & 19 & 4,79 & 86 & 21,66 \\
\hline TOTAL & 144 & 36,27 & 88 & 22,17 & 82 & 20,65 & 83 & 20,91 & 397 & 100 \\
\hline
\end{tabular}

La Tabla 7 según la prueba de chi cuadrado $\left(X^{2}=5.62\right)$ muestra que el grado de ansiedad y la Retractación de afrontamiento no presenta relación estadística significativa ( $\mathrm{P}>0.05)$. Asimismo, se observa que el 23.93\% de los estudiantes universitarios de la región
Moquegua con ansiedad mínima presentan retracción de afrontamiento dependiendo de las circunstancias, mientras que el $4.79 \%$ de los estudiantes con ansiedad severa presentan frecuentemente este tipo de afrontamiento. 
De la misma manera, en la Tabla 8 se muestra la relación entre el grado de ansiedad/estrés consecuencia de la Pandemia y la búsqueda de soporte social en los alumnos de la universidad.

Tabla 8. Relación entre el grado de ansiedad/estrés a consecuencia de la pandemia y la búsqueda de soporte social en los alumnos de la Universidad Nacional de Moquegua.

\begin{tabular}{|c|c|c|c|c|c|c|c|c|c|c|}
\hline \multirow{3}{*}{$\begin{array}{l}\text { Búsqueda de } \\
\text { soporte social }\end{array}$} & \multicolumn{8}{|c|}{ ANSIEDAD } & \multirow{2}{*}{\multicolumn{2}{|c|}{ TOTAL }} \\
\hline & \multicolumn{2}{|c|}{ Mínima } & \multicolumn{2}{|c|}{ Leve } & \multicolumn{2}{|c|}{ Moderada } & \multicolumn{2}{|c|}{ Severa } & & \\
\hline & №. & $\%$ & №. & $\%$ & №. & $\%$ & №. & $\%$ & №. & $\%$ \\
\hline Pocas veces & 34 & 8,56 & 29 & 7,30 & 26 & 6,55 & 33 & 8,31 & 122 & 30,73 \\
\hline Dep. circunstancias & 43 & 10,83 & 22 & 5,54 & 23 & 5,79 & 21 & 5,29 & 109 & 27,46 \\
\hline Frecuentemente & 67 & 16,88 & 37 & 9,32 & 33 & 8,31 & 29 & 7,30 & 166 & 41,81 \\
\hline TOTAL & 144 & 36,27 & 88 & 22,17 & 82 & 20,65 & 83 & 20,91 & 397 & 100 \\
\hline
\end{tabular}

La Tabla 8 muestra los resultados obtenidos y según la prueba de chi cuadrado $\left(X^{2}=7.14\right)$ muestra que el grado de ansiedad y la búsqueda de soporte social no presenta relación estadística significativa $(\mathrm{P}>0.05)$. Igualmente se observa que el $16.88 \%$ de los estudiantes universitarios de la región Moquegua con ansiedad mínima presentan frecuentemente búsqueda de soporte social, mientras que el $8.31 \%$ de los estudiantes con ansiedad severa pocas veces tienen este tipo de afrontamiento.

Asimismo, en la Tabla 9 se muestra la relación entre el grado de ansiedad/estrés a consecuencia de la pandemia y la búsqueda de soporte emocional en los alumnos de la Universidad Nacional de Moquegua.

Tabla 9. Relación entre el grado de ansiedad/estrés a consecuencia de la pandemia y la búsqueda de soporte emocional en los alumnos de la Universidad Nacional de Moquegua.

\begin{tabular}{|c|c|c|c|c|c|c|c|c|c|c|}
\hline \multirow{3}{*}{$\begin{array}{l}\text { Búsqueda de } \\
\text { soporte emocional }\end{array}$} & \multicolumn{8}{|c|}{ ANSIEDAD } & \multirow{2}{*}{\multicolumn{2}{|c|}{ TOTAL }} \\
\hline & \multicolumn{2}{|c|}{ Mínima } & \multicolumn{2}{|c|}{ Leve } & \multicolumn{2}{|c|}{ Moderada } & \multicolumn{2}{|c|}{ Severa } & & \\
\hline & №. & $\%$ & №. & $\%$ & №. & $\%$ & №. & $\%$ & №. & $\%$ \\
\hline Pocas veces & 48 & 12,09 & 34 & 8,56 & 36 & 9,07 & 43 & 10,83 & 161 & 40,55 \\
\hline Dep. circunstancias & 47 & 11,84 & 30 & 7,56 & 21 & 5,29 & 22 & 5,54 & 120 & 30,23 \\
\hline Frecuentemente & 49 & 12,34 & 24 & 6,05 & 25 & 6,30 & 18 & 4,53 & 116 & 29,22 \\
\hline TOTAL & 144 & 36,27 & 88 & 22,17 & 82 & 20,65 & 83 & 20,91 & 397 & 100 \\
\hline & & $=9.3$ & & $>0.05$ & & $=0$. & & & & \\
\hline
\end{tabular}

La Tabla 9 según la prueba de chi cuadrado $\left(\mathrm{X}^{2}=9.33\right)$ muestra que el grado de ansiedad y la búsqueda de soporte emocional no presenta relación estadística significativa ( $\mathrm{P}>0.05$ ).Asimismo, se observa que el $10.83 \%$ de los estudiantes universitarios de la región
Moquegua con ansiedad severa pocas veces presentan búsqueda de soporte emocional, mientras que el $12.34 \%$ de los estudiantes con ansiedad mínima frecuentemente tienen este tipo de afrontamiento. 
De la misma manera en la Tabla 10 se evidencia la relación entre el grado de ansiedad/estrés a consecuencia de la pandemia y la interpretación positiva del desarrollo personal en los alumnos de la Universidad Nacional de Moquegua.

Tabla 10. Relación entre el grado de ansiedad/estrés a consecuencia de la pandemia y la reinterpretación positiva del desarrollo personal en los alumnos de la Universidad Nacional de Moquegua.

\begin{tabular}{|c|c|c|c|c|c|c|c|c|c|c|}
\hline \multirow{3}{*}{$\begin{array}{c}\text { Reinterpretación } \\
\text { positiva desarrollo } \\
\text { personal }\end{array}$} & \multirow{2}{*}{\multicolumn{2}{|c|}{ Mínima }} & \multicolumn{4}{|c|}{ ANSIEDAD } & \multirow{2}{*}{\multicolumn{2}{|c|}{ Severa }} & \multirow{2}{*}{\multicolumn{2}{|c|}{ TOTAL }} \\
\hline & & & & eve & Mo & erada & & & & \\
\hline & №. & $\%$ & №. & $\%$ & №. & $\%$ & №. & $\%$ & №. & $\%$ \\
\hline Pocas veces & 7 & 1,76 & 6 & 1,51 & 6 & 1,51 & 11 & 2,77 & 30 & 7,56 \\
\hline Dep. circunstancias & 26 & 6,55 & 24 & 6,05 & 27 & 6,80 & 31 & 7,81 & 108 & 27,20 \\
\hline Frecuentemente & 111 & 27,96 & 58 & 14,61 & 49 & 12,34 & 41 & 10,33 & 259 & 65,24 \\
\hline TOTAL & 144 & 36,27 & 88 & 22,17 & 82 & 20,65 & 83 & 20,91 & 397 & 100 \\
\hline
\end{tabular}

En la Tabla 10 según la prueba de chi cuadrado $\left(X^{2}=20.24\right)$ muestra que el grado de ansiedad y la reinterpretación positiva del desarrollo personal presentan relación estadística significativa $(\mathrm{P}<0.05)$.Asimismo se observa que el $27.96 \%$ de los estudiantes universitarios de la región Moquegua con ansiedad mínima frecuentemente tienen reinterpretación positiva del desarrollo personal, mientras que el $6.80 \%$ de los estudiantes con ansiedad moderada tienen este tipo de afrontamiento dependiendo de las circunstancias.

En este sentido en la Tabla 11 se muestra la correlación entre el grado de ansiedad/estrés a consecuencia de la pandemia y la reinterpretación positiva del desarrollo personal en los alumnos de la Universidad Nacional de Moquegua.

Tabla 11. Correlación entre el grado de ansiedad/estrés a consecuencia de la pandemia y la reinterpretación positiva del desarrollo personal en los alumnos de la Universidad Nacional de Moquegua.

\begin{tabular}{ccccc}
\hline & Correlaciones & & $\begin{array}{c}\text { Reinterpretación } \\
\text { positiva y desarrollo } \\
\text { personal }\end{array}$ \\
\hline $\begin{array}{c}\text { Rho de } \\
\text { Spearman }\end{array}$ & Ansiedad & Coeficiente de correlación & 1,000 &, $809^{* *}$ \\
& & Sig. (bilateral) & - &, 000 \\
& Reinterpretación & Coeficiente de correlación &, $809^{* *}$ & 397 \\
& positiva y desarrollo & Sig. (bilateral) &, 000 & 1,000 \\
& personal & N & 397 &. \\
\hline
\end{tabular}

**. La correlación es significativa en el nivel 0,01 (bilateral). 
Según la correlación de Spearman (Rho=.415) existe una correlación muy alta entre el nivel de ansiedad y la reinterpretación positiva del desarrollo personal $(\mathrm{P}<0.05)$.
Seguidamente se muestra la tabla 12 donde se evidenció la relación entre el grado de ansiedad/estrés a consecuencia de la pandemia y la aceptación en los alumnos de la Universidad Nacional de Moquegua.

Tabla 12. Relación entre el grado de ansiedad/estrés a consecuencia de la pandemia y la aceptación en los alumnos de la Universidad Nacional de Moquegua.

\begin{tabular}{|c|c|c|c|c|c|c|c|c|c|c|}
\hline \multirow{3}{*}{ Aceptación } & \multicolumn{8}{|c|}{ ANSIEDAD } & \multirow{2}{*}{\multicolumn{2}{|c|}{ TOTAL }} \\
\hline & \multicolumn{2}{|c|}{ Mínima } & \multicolumn{2}{|c|}{ Leve } & \multicolumn{2}{|c|}{ Moderada } & \multicolumn{2}{|c|}{ Severa } & & \\
\hline & №. & $\%$ & №. & $\%$ & №. & $\%$ & №. & $\%$ & №. & $\%$ \\
\hline Pocas veces & 11 & 2,77 & 4 & 1,01 & 4 & 1,01 & 8 & 2,02 & 27 & 6,80 \\
\hline Dep. circunstancias & 66 & 16,62 & 35 & 8,82 & 33 & 8,31 & 34 & 8,56 & 168 & 42,32 \\
\hline Frecuentemente & 67 & 16,88 & 49 & 12,34 & 45 & 11,34 & 41 & 10,33 & 202 & 50,88 \\
\hline TOTAL & 144 & 36,27 & 88 & 22,17 & 82 & 20,65 & 83 & 20,91 & 397 & 100 \\
\hline & & $X^{2}=4.13$ & & $P>0.05$ & & $P=0.6$ & & & & \\
\hline
\end{tabular}

La Tabla 12 según la prueba de chi cuadrado $\left(\mathrm{X}^{2}=4.13\right)$ muestra que el grado de ansiedad y la aceptación no presentó relación estadística significativa $(\mathrm{P}>0.05)$.Asimismo, se observa que el $16.88 \%$ de los estudiantes universitarios de la región Moquegua con ansiedad mínima frecuentemente presentan el estilo de afrontamiento de aceptación, mientras que el $8.82 \%$ de los estudiantes con ansiedad leve tienen este tipo de afrontamiento dependiendo de las circunstancias.

De la misma manera en la Tabla 13 se muestra la relación entre el grado de ansiedad/estrés a consecuencia de la pandemia y el análisis de emociones en los alumnos de la Universidad Nacional de Moquegua.

Tabla 13. Relación entre el grado de ansiedad/estrés a consecuencia de la pandemia y el análisis de emociones en los alumnos de la Universidad Nacional de Moquegua.

\begin{tabular}{|c|c|c|c|c|c|c|c|c|c|c|}
\hline \multirow{3}{*}{$\begin{array}{l}\text { Análisis de } \\
\text { emociones }\end{array}$} & \multicolumn{8}{|c|}{ ANSIEDAD } & \multirow{2}{*}{\multicolumn{2}{|c|}{ TOTAL }} \\
\hline & \multicolumn{2}{|c|}{ Mínima } & \multicolumn{2}{|c|}{ Leve } & \multicolumn{2}{|c|}{ Moderada } & \multicolumn{2}{|c|}{ Severa } & & \\
\hline & №. & $\%$ & №. & $\%$ & №. & $\%$ & №. & $\%$ & №. & $\%$ \\
\hline Pocas veces & 90 & 22,67 & 53 & 13,35 & 44 & 11,08 & 50 & 12,59 & 237 & 59,70 \\
\hline Dep. circunstancias & 45 & 11,34 & 32 & 8,06 & 29 & 7,30 & 25 & 6,30 & 131 & 33,00 \\
\hline Frecuentemente & 9 & 2,27 & 3 & 0,76 & 9 & 2,27 & 8 & 2,02 & 29 & 7,30 \\
\hline TOTAL & 144 & 36,27 & 88 & 22,17 & 82 & 20,65 & 83 & 20,91 & 397 & 100 \\
\hline & & $X^{2}=5.6$ & & $P>0.05$ & & $P=0.4$ & & & & \\
\hline
\end{tabular}


La Tabla 13 según la prueba de chi cuadrado $\left(X^{2}=5.66\right)$ muestra que el grado de ansiedad $\mathrm{y}$ el análisis de emociones no presentan relación estadística significativa $(\mathrm{P}>0.05)$.De esta manera se observa que el $22.67 \%$ de los estudiantes universitarios de la región Moquegua con ansiedad mínima pocas veces analizan sus emociones, mientras que el
$7.30 \%$ de los estudiantes con ansiedad moderada tienen este estilo de afrontamiento dependiendo de las circunstancias.

En este sentido la Tabla 14 refleja la relación entre el grado de ansiedad/estrés a consecuencia de la pandemia y la negación en los alumnos de la Universidad Nacional de Moquegua.

Tabla 14. Relación entre el grado de ansiedad/estrés a consecuencia de la pandemia y la negación en los alumnos de la Universidad nacional de Moquegua.

\begin{tabular}{|c|c|c|c|c|c|c|c|c|c|c|}
\hline \multirow{3}{*}{ Negación } & \multicolumn{8}{|c|}{ ANSIEDAD } & \multirow{2}{*}{\multicolumn{2}{|c|}{ TOTAL }} \\
\hline & \multicolumn{2}{|c|}{ Mínima } & \multicolumn{2}{|c|}{ Leve } & \multicolumn{2}{|c|}{ Moderada } & \multicolumn{2}{|c|}{ Severa } & & \\
\hline & №. & $\%$ & №. & $\%$ & №. & $\%$ & №. & $\%$ & №. & $\%$ \\
\hline Pocas veces & 91 & 22,92 & 39 & 9,82 & 28 & 7,05 & 29 & 7,30 & 187 & 47,10 \\
\hline Dep. circunstancias & 37 & 9,32 & 41 & 10,33 & 34 & 8,56 & 41 & 10,33 & 153 & 38,54 \\
\hline Frecuentemente & 16 & 4,03 & 8 & 2,02 & 20 & 5,04 & 13 & 3,27 & 57 & 14,36 \\
\hline TOTAL & 144 & 36,27 & 88 & 22,17 & 82 & 20,65 & 83 & 20,91 & 397 & 100 \\
\hline
\end{tabular}

La Tabla 14 según la prueba de chi cuadrado $\left(\mathrm{X}^{2}=32.56\right)$ demostró que el grado de ansiedad y la negación presentan relación estadística significativa $(\mathrm{P}<0.05)$. Según la correlación de Spearman (Rho=.851) existe una correlación muy alta entre las variables. De esta forma se observa que el $22.92 \%$ de los estudiantes universitarios de la región Moquegua con ansiedad mínima pocas veces tienen el estilo de negación, mientras que el $10.33 \%$ de los estudiantes con ansiedad severa tienen este estilo de afrontamiento dependiendo de las circunstancias.

Seguidamente en la Tabla 15 se muestra la relación entre el grado de ansiedad/estrés a consecuencia de la pandemia y las conductas inadecuadas en los alumnos de la Universidad Nacional de Moquegua.

Tabla 15. Relación entre el grado de ansiedad/estrés a consecuencia de la pandemia y las conductas inadecuadas en los alumnos de la Universidad Nacional de Moquegua.

\begin{tabular}{|c|c|c|c|c|c|c|c|c|c|c|}
\hline \multirow{3}{*}{$\begin{array}{l}\text { Conducta } \\
\text { inadecuada }\end{array}$} & \multicolumn{8}{|c|}{ ANSIEDAD } & \multirow{2}{*}{\multicolumn{2}{|c|}{ TOTAL }} \\
\hline & \multicolumn{2}{|c|}{ Mínima } & \multicolumn{2}{|c|}{ Leve } & \multicolumn{2}{|c|}{ Moderada } & \multicolumn{2}{|c|}{ Severa } & & \\
\hline & №. & $\%$ & №. & $\%$ & №. & $\%$ & №. & $\%$ & №. & $\%$ \\
\hline Pocas veces & 113 & 28,46 & 57 & 14,36 & 40 & 10,08 & 47 & 11,84 & 257 & 64,74 \\
\hline Dep. circunstancias & 23 & 5,79 & 27 & 6,80 & 34 & 8,56 & 33 & 8,31 & 117 & 29,47 \\
\hline Frecuentemente & 8 & 2,02 & 4 & 1,01 & 8 & 2,02 & 3 & 0,76 & 23 & 5,79 \\
\hline TOTAL & 144 & 36,27 & 88 & 22,17 & 82 & 20,65 & 83 & 20,91 & 397 & 100 \\
\hline
\end{tabular}


La Tabla 15 según la prueba de chi cuadrado $\left(\mathrm{X}^{2}=27.34\right)$ muestra que el grado de ansiedad y la conducta inadecuada presentan relación estadística significativa $(\mathrm{P}<0.05)$. Según la correlación de Spearman (Rho=.718) existe una correlación alta entre las variables. De la misma forma se observa que el $28.46 \%$ de los estudiantes universitarios de la región Moquegua con ansiedad mínima pocas veces tienen conductas inadecuadas, mientras que el $8.31 \%$ de los estudiantes con ansiedad severa tienen este estilo de afrontamiento dependiendo de las circunstancias.

Finalmente en la Tabla 16 se muestra la relación entre el grado de ansiedad/estrés a consecuencia de la pandemia y la distracción en los alumnos de la Universidad Nacional de Moquegua.

Tabla 16. Relación entre el grado de ansiedad/estrés a consecuencia de la pandemia y la distracción en los alumnos de la Universidad Nacional de Moquegua.

\begin{tabular}{|c|c|c|c|c|c|c|c|c|c|c|}
\hline \multirow{3}{*}{ Distracción } & \multicolumn{8}{|c|}{ ANSIEDAD } & \multirow{2}{*}{\multicolumn{2}{|c|}{ TOTAL }} \\
\hline & & lima & & eve & Mo & erada & & jera & & \\
\hline & №. & $\%$ & №. & $\%$ & №. & $\%$ & №. & $\%$ & №. & $\%$ \\
\hline Pocas veces & 42 & 10,58 & 17 & 4,28 & 19 & 4,79 & 25 & 6,30 & 103 & 25,94 \\
\hline Dep. circunstancias & 81 & 20,40 & 57 & 14,36 & 42 & 10,58 & 51 & 12,85 & 231 & 58,19 \\
\hline Frecuentemente & 21 & 5,29 & 14 & 3,53 & 21 & 5,29 & 7 & 1,76 & 63 & 15,87 \\
\hline TOTAL & 144 & 36,27 & 88 & 22,17 & 82 & 20,65 & 83 & 20,91 & 397 & 100 \\
\hline
\end{tabular}

La Tabla 16 según la prueba de chi cuadrado $\left(\mathrm{X}^{2}=12.39\right)$ muestra que el grado de ansiedad y la distracción presentan relación estadística significativa $(\mathrm{P}<0.05)$. Según la correlación de Spearman (Rho=.718) existe una correlación muy baja entre las variables. De este modo se observa que el $20.40 \%$ de los estudiantes universitarios de la región Moquegua con ansiedad mínima tienen distracciones dependiendo de las circunstancias, mientras que el $5.29 \%$ de los estudiantes con ansiedad moderada tienen este estilo de afrontamiento frecuentemente.

\section{Discusión}

En referencia a las características sociodemográficas el $41.56 \%$ de los estudiantes universitarios pertenecen a la escuela profesional de Ingeniería de minas, seguido del $12.34 \%$ de estudiantes de la escuela de gestión pública y desarrollo social, mientras que solo el $3.53 \%$ son de ingeniería pesquera, el $77.3 \%$ de los estudiantes tienen entre 18 a 28 años, seguido del $16.6 \%$ de estudiantes menores de 18 años, el 4.5\% tienen entre 29 a 39 años, mientras que solo el $1.5 \%$ tienen 40 años o más, el $50.88 \%$ son de sexo masculino, mientras que el $49.12 \%$ de los estudiantes son de sexo femenino y por último el $97.48 \%$ de los estudiantes son solteros, mientras que el $2.52 \%$ de los estudiantes son casados.

De acuerdo a la escala de Beck el 36.27\% de los estudiantes universitarios de la región Moquegua presentan niveles mínimos o casi inexistentes de ansiedad, seguido del $22.17 \%$ de estudiantes con ansiedad leve, el 20.91\% tienen ansiedad severa, mientras que el $20.65 \%$ presentan ansiedad moderada. Investigaciones similares sostiene que los 
estudiantes universitarios experimentan angustia contribuida por la incertidumbre de la interrupción abrupta del semestre, además de la ansiedad causada por el cierre de la escuela y universidades. A medida que más universidades hacen la transición al aprendizaje remoto después de las vacaciones de primavera, algunos estudiantes sufren de mala salud mental debido a la interrupción de la rutina académica (Agnew, Poole, y Khan, 2019). Muchos estudiantes tienen que dejar sus proyectos de investigación y pasantías cuando las universidades los evacuaron del campus. Además, las interrupciones de sus proyectos de investigación y pasantías ponen en peligro su programa de estudios, retrasan su graduación y socavan su competitividad en el mercado laboral, lo que a su vez alimenta la ansiedad entre los estudiantes universitarios. También pueden tener problemas con el costo de regresar a casa y administrar sus pertenencias. La UNESCO estima que el cierre de las instituciones educativas debido a la pandemia está afectando a la mitad de los estudiantes del mundo: 890 millones en 114 países. La enseñanza en línea ahora es una nueva rutina para algunos estudiantes, pero presenta desafíos importantes. No todos los alumnos tienen acceso a este tipo de educación, considerando la desigualdad social en muchas naciones (UNESCO, 2020; FVG, 2020). Según el monitoreo de la UNESCO, más de 160 países implementaron cierres a nivel nacional, lo que impactó a más del $87 \%$ de la población estudiantil del mundo. Varios otros países implementaron cierres escolares localizados; En caso de que estos cierres lleguen a todo el país, millones de estudiantes adicionales experimentarán interrupciones educativas (UNESCO, 2020).

Con respecto a los tipos de afrontamiento frente al estrés el $41.81 \%$ de los estudiantes universitarios presentan frecuentemente afrontamiento directo, el $50.88 \%$ suelen presentar con frecuencia el estilo de aceptación, el $40.55 \%$ pocas veces presenta búsqueda de soporte emocional, el 58.19\% presentan distracción dependiendo de las circunstancias, mientras que el $18.14 \%$ pocas veces tienen planificación de actividades. Muchas universidades decidieron suspender las clases en persona y evacuar a los estudiantes para responder a las preocupaciones cada vez más intensas en torno a COVID19. Esta acción puede tener consecuencias psicológicas negativas entre los estudiantes universitarios. Por ejemplo, los estudiantes educación superior a menudo experimentan emociones negativas compuestas durante el "cierre" de la universidad (Van Bortel et al. 2020).

Algunos estudiantes que consideran que el campus es acogedor. Pero albergan sentimientos intensos como la frustración, la ansiedad y la traición. Algunos pueden luchar con la soledad y el aislamiento mientras se refugia en el lugar debido a desconexiones de amigos y parejas. Para aquellos que reciben servicios de asesoramiento en el campus, ya que no pueden acceder a los servicios de asesoramiento presencial, lo que exacerba sus síntomas psicológicos y aumenta el riesgo de suicidio y abuso de sustancias de algunos estudiantes.

Después de aplicar la prueba de chi cuadrado se determinó que los niveles de ansiedad están relacionados significativamente con los siguientes tipos de afrontamiento: supresión de actividades, reinterpretación positiva y desarrollo personal, negación, conducta inadecuada y distracción. El 15.62\% de los estudiantes universitarios de la región Moquegua con ansiedad mínima pocas veces presentan el 
estilo de afrontamiento de supresión de actividades, mientras que el $10.33 \%$ de los estudiantes con ansiedad moderada presentan este tipo de afrontamiento dependiendo de las circunstancias. El 27.96\% de los con ansiedad mínima frecuentemente tienen reinterpretación positiva del desarrollo personal, mientras que el $6.80 \%$ de los estudiantes con ansiedad moderada tienen este tipo de afrontamiento dependiendo de las circunstancias. La COVID-19 y sus efectos acompañantes continuarán impactando profundamente la salud mental y el bienestar universitario; a consecuencia la salud mental desempeña un papel crucial en la lucha contra la epidemia (Zhai y Du, 2020). Es imperativo para las universidades crear conciencia sobre las necesidades y preocupaciones de salud mental de los estudiantes, y capacitar a sus estudiantes para buscar ayuda y apoyo durante este desastre biológico. Los estudiantes universitarios deben adaptar las estrategias de afrontamiento para satisfacer sus necesidades específicas y promover su capacidad de recuperación psicológica. Los esfuerzos considerables realizados por las universidades deberían dedicarse a ayudar a los estudiantes a prosperar en esta crisis. Con la experiencia obtenida apoyando a los estudiantes en esta pandemia, las universidades estarán bien posicionadas para ayudar a los estudiantes universitarios a mantenerse en mente, cuerpo y espíritu durante otros tiempos difíciles.

\section{CONCLUSIONES}

Esta investigación se realizó con el objetivo de evaluar el aislamiento domiciliario a consecuencia del coronavirus y su repercusión en la salud mental del estudiante universitario de la región
Moquegua 2020, en este sentido se pudo determinar que de acuerdo a los resultados obtenidos el aislamiento domiciliario tiene repercusión en la capacidad que tiene el alumno de afrontar las situaciones en cuanto al tema universitario, sin embargo a pesar de esto muchos estudiantes logran afrontar las situaciones, planificando y creando estrategias que le permitan coordinar y poder ejecutar sus actividades, en ocasiones asumen la situación y buscan ayuda emocional para poder afrontar la ansiedad que están presentando, y así adaptarse a las exigencias de esta nueva normalidad.

\section{REFERENCIAS}

Agnew, M., Poole, H., y Khan, A. (2019). Fall break fallout: Exploring student perceptions of the impact of an autumn break on stress. Student Success, 10(3), 4554

Barraza, A., y Quiñonez, J. (2007). El estrés académico en alumnos de educación media superior: Un estudio comparativo. Investigación Educativa

Bunevicius, A., Katkute, A., y Bunevicius, R. (2008). Symptoms of anxiety and depression in medical students and in humanities students: relationship with big-five personality dimensions and vulnerability to stress. International Journal of Social Psychiatry, 54(6), 494501

Caldera, J., y Pulido, B. (2007). Niveles de estrés y rendimiento académico en estudiantes de la carrera de Psicología del Centro Universitario de Los Altos. Revista de Educación y Desarrollo, 7

Carver, C., Scheier, M., y Weintraub, J. (1989). Assessing coping strategies: A theoretically based approach. Journal of Personality and Social Psychology, 56(2), 267-283

Chinese Center for Disease Control and Prevention. (2020). Home/COVID19. 
Recuperado el mayo de 2021, de http://www.chinacdc.cn/en/COVID19/

DECRETO SUPREMO $\mathrm{N}^{\circ}$ 044-2020-PCM. (2020). Decreto Supremo que declara Estado de Emergencia Nacional por las graves circunstancias que afectan la vida de la Nación a consecuencia del brote del COVID-19. Lima, Perú. El Peruano

DECRETO SUPREMO No 116-2020-PCM. (2020). Decreto Supremo que establece las medidas que debe observar la ciudadanía en la Nueva Convivencia Social y prorroga el Estado de Emergencia Nacional por las graves circunstancias que afectan la vida de la Nación a consecuencia del COVID-19. Perú: El Peruano. Diario Oficial del Bicentenario

FVG. (2020). Webinar discusses education challenges during coronavirus pandemic. Recuperado el 03 de Mayo de 2021, de https://portal.fgv.br/en/news/webinardiscusses-education-challengesduringcoronavirus-pandemic

Guan, W., Ni, Z., Hu, Y., Liang, W., Ou, C., He, J., $y$ otros. (2020). Clinical characteristics of coronavirus disease 2019 in China. $N$. Engl. J. Med. 382, 1708-1720

Hudd, S., Dumlao, J., Erdmann-Sager, D., Murray, D., Phan, E., Soukas, N., y otros. (2000). Stress at college: effects on health habits, health status and self-esteem. College Student Journal, 34(2), 217-227

Li, Q., Guan, X., Wu, P., Wang, X., Zhou, L., Tong, Y., y otros. (2020). Early transmission dynamics inWuhan, China, of novel coronavirus-infected pneumonia. N. Engl. J. Med. 382 , 1199-1207

Martínez, E., y Diaz, D. (2007). Una aproximación psicosocial al estrés escolar Educación y Educadores. Universidad de La Sabana Cundinamarca 10(2)

Misra, R., Crist, M., y Burant, C. (2003). Relationships among life stress, social support, academic stressors and reactions to stressors of internacional students in the United States. International Journal of Stress Management, 10(2), 137-157

Morales, E. (2011). La ansiedad en el ámbito universitario. Revista Griot. 4 (1), 35-48

Nieto, J., y Nieto, L. (2006). Cómo evitar o superar el estrés. Educar: Madrid, España

Pan, X., Chen, D., Xia, Y., Wu, X., Li, T., Ou, X., y otros. (2020). Asymptomatic cases in a family cluster with SARS-CoV-2 infection. Lancet Infect Dis. 20(4), 410-411

Tian, F., Li, H., Tian, S., Yang, J., Shao, J., y Tian, C. (2020). Psychological symptoms of ordinary Chinese citizens based on SCL-90 during the level I emergency response to COVID-19. Psychiatry research, 288:112992

UNESCO. (2020). COVID-19 educational disruption and response. Recuperado el 04 de Mayo de 2021, de https://en.unesco.org/themes/educationemergencies/coronavirus-school-closures

Van Bortel, T., Basnayake, A., Wurie, F., Jambai, M., Koroma, A., Muana, A., y otros. (2020). Psychosocial effects of an Ebola outbreak at individual, community and international levels. Bull. World Health Organization

World Health Organization. (2020). Coronavirus Disease 2019 (COVID-19) Situation Report-57. Recuperado el mayo de 2021, de https://www.who.int/docs/defaultsource /coronaviruse/situationreports/2020031 7-sitrep-57-covid-19.pdf

Zhai, Y., y Du, X. (2020). Addressing collegiate mental health amid COVID-19 pandemic. Psychiatry Research, 288, 113003

Zhu, N., Zhang, D., Wang, W., Li, X., Yang, B., Song, J., y otros. (2019). A novel coronavirus from patients with pneumonia in China. N. Engl. J. Med. 382, 727-733 